\title{
Organizational structure and innovative performances of the firm: A dynamic model*
}

\author{
FRANCIS MUNIER**
}

\begin{abstract}
The aim of this article is to propose an analytical basis for the hypothesis according to which interactions between individuals increase innovative performances of the firm. The model is based on the mathematical theory of viability, which enables comparison between different dynamics of evolution of organizational structures.
\end{abstract}

Classification Codes: D22, C6.

\section{Introduction}

The aim of this article is to demonstrate analytically the central hypothesis of the model of organizational knowledge creation developed by Nonaka $(1990,1994,1995)$. This hypothesis supposes that interactions between individuals in a firm promote collective learning and increase innovative performances of the firm. We propose to describe, in terms of mathematical metaphors, different forms of an organizational structure, each describing innovative behavior, in order to compare their dynamical evolution. Either the organization authorizes interactions between the individuals, or the process of innovation is summarized in the individual behaviors in the form of a message. We show that the dynamics of evolution of these two forms of regulation is identical. In other words, we show that the dynamics of innovative behavior where agents interact is the same as the dynamic where the innovative performances of the firm are included in individual's contribution.

The originality of our approach rests on the assumption according to which the firm does not adopt optimizing behavior. We pose the hypothesis of nonstochastic uncertainty (contingent uncertainty) to explain that the innovative performances of the firm do not constitute an objective to be reached. Competencies held by the individuals within the

\footnotetext{
* This work benefited from the financial support of the CNRS programme upon « Les enjeux économiques de l'innovation ». The author gratefully thanks in particular Jean-Pierre Aubin, and also Jean-Alain Héraud and Hubert Stahn for their remarks and suggestions and is obviously alone liable for mistakes or omissions. ** BETA, UMR du CNRS n ${ }^{\circ} 7522$, Université Louis Pasteur, 61 avenue de la Forêt-Noire, 67085 Strasbourg, France. E-mail : munier@cournot.u-strasbg.fr

Keywords: Viability, innovation, interaction, learning.
} 
firm guide the action and confer an attitude of satisfacing according to the innovative performances.

To describe these hypotheses mathematically, we base our analysis on the recent developments of the viability theory (Aubin, 1991; 1997). Schematically, this theory sets out to explain the evolution of a system described by a given nondeterministic dynamics and viability constraints, to reveal the concealed regulation laws which allow the system to be regulated and provide selection mechanisms for implementing them. A terminology will help to clarify the basic principles. A system is described by two classes of variables: state variables and regulation variables (or regulees). The former corresponds to the components of the state of the system and can evolve under the impetus of agents. The states evolve according to the regulee, defined as a message detected by the system.

In our framework, individuals' contributions to the innovative process are the state variable. The regulee defines the innovative performances of the firm summarized as Information Redundancy (Nonaka, 1990). The competencies of the individuals are the viability constraints of the model.

In the first part, we will present the basis of the viability theory. In the second part, we will examine the model of organizational knowledge creation applied to innovation themes. In the third and last part, we will describe, mathematically, different organizational structures for which we will explain the problem of viability in order then to demonstrate the role of individual interactions in the process of organizational knowledge creation put forward by Nonaka $(1990,1994,1995)$.

\section{Some glimpses of the viability theory}

The main object of the viability theory ${ }^{1}$ is to explain the evolution of a system described by given nondeterministic dynamics and viability constraints, to reveal the concealed regulation laws that allow the system to be regulated, and provide selection mechanisms for implementing them.

A common definition of viability is something that can be adapted to constraints in order to be able to live. Viability theory rejects therefore the teleological principle of optimization to focus on viability constraints, on the adaptation of the system. At each moment, a state of the system must respect the viability constraints.

A system is described by state variables and regulation variables. The former correspond to components of the state of the system and can evolve under the impulse of agents. The states evolve according to the regulee, defined as a regulating control or a message detected by the system.

A dynamic law associated to this state and to all regulees defines the velocity of evolution of the state. A second law, called the a priori retroaction, describes the constraints to which the regulees are subjected according to the current or past state of the system. It

\footnotetext{
${ }^{1}$ We refer to Aubin $(1991,1997)$. The reader may also refer to Aubin and Foray (1998) for an application of viability theory in the field of network economy.
} 
determines all the available regulees, thus clarifying a form of diversity, in the framework of a situation whereby a contingent uncertainty ${ }^{2}$ prevails.

Viability theory therefore constitutes a study of the dynamic evolution of a system beyond contingent uncertainty and under viability constraints. The set of states of the system which comply with viability constraints is referred to as constrained set. A viable state is an element of this set. A viable evolution is an evolution, which verifies, at each moment, the viability constraints.

This consistency between the dynamics of the system and the viability constraints is important. The initial question, to which the viability theory provides some answers, can be expressed as follows: with each viable state of the macrosystem as an initial state (element of the constrained set), is there at least one viable evolution? ( $c f$. Def. 4.1.2 in Aubin, 1997, p. 143). The mathematical resolution of compatibility of constraints and of dynamics of a system is made using the regulation law. This law results from the basic viability theorem, which consists in considering that a viable evolution is governed by a viable regulee, obtained through the intermediary of the regulation map.

The system is also characterized by three basic principles. On the one hand, its evolution is nondeterministic in the sense that the system is subjected to a contingent uncertainty, and that the existence of a multitude of evolutions according to the different regulees is possible. It also illustrates the idea of flexibility if one considers that it is possible to develop different possible regulees where the environment does not provide for this. On the other hand, the system is subject to viability constraints, which the state of the system must respect "on pain of death". These constraints limit the evolution of the system and determine the domain of constraint in which this evolution must imperatively occur. The third basic principle suggests that the evolution of the system complies with a principle of inertia according to which the regulee evolves only if viability conditions are questioned. If the evolution happens as slowly as possible, this is called the principle of heavy evolution.

The notion of the viability domain is introduced in order to avoid a situation where all the viable regulees are empty in at least one state of the system. The distinction between a constrained set and a viable domain is crucial in the sense that it allows the notion of consistency between a dynamics and the constraints to which it is confronted. The theorem of the theory of viability is based on this necessary compatibility: a constrained set is compatible with a macrosystem if and only if it is a viability domain of that macrosystem ( $c f$. Th. 4.1.5. in Aubin, 1997, p. 145).

The mathematical theory of viability ${ }^{3}$ uses tools from set-valued analysis such as setvalued maps. It consists in using a set-valued map $C$, where we associate $x \in E$ with a set (possible empty) $C(x) \subset F$. On the contrary, in the single-valued analysis, each element $x \in E$ is a unique element $f(x) \in F$.

\footnotetext{
${ }^{2}$ Contingent uncertainty infers that each regulee has the same chance as the others to be the one chosen in the determination of the regulation map.

${ }^{3}$ The first theorem in the framework of differential inclusion was defined by Bebernes and Schuur (1970) and Haddad (1981).
} 
This approach enables the treatment of nondeterministic and non-stochastic dynamic models and can also take into account regulation parameters. Its relevance is to widen the spectrum of the study for a solution of the differential equation by replacing it with the notion of differential inclusion.

The advantage of using differential inclusion is thus, on the one hand, to allow the treatment of certain random forms, notably the random form defined by nonstochastic uncertainty. On the other hand, the differential inclusions fit on the case of complex systems, which differ from each other by the absence of control and a variety of possible dynamics. These are short-sighted systems that do not attempt to attain teleological objectives (satisfacing behavior). In contrast to the optimization, only minimal satisfaction requirements are to be respected. The differential inclusion is described by $x^{\prime}(t) \in F(x(t))$, where when at each moment $t$ the state of the system is $x(t)$, the set $F$ determines the set of possible velocities for this state.

The presentation of the fundamental characteristics of viability theory gives some explaining keys to better understand the model that we will present in the third section. Before that, we will now examine, conceptually, the model of organizational knowledge creation developed by Nonaka.

\section{Individual interaction and creation of organizational knowledge}

Nonaka $(1990,1994,1995)$ has developed a theory of the creation of organizational knowledge in the firm. The focal point concerns the importance of the interaction between individuals and also between tacit and codified knowledge. He suggests that a permanent dialogue between these actors is the main source of new knowledge. We will introduce the basic concepts of this theory in order to explain the idea of commitment, keystone to the process of the creation of knowledge. We will also explain the concept of information redundancy necessary to understand the organizational knowledge creation to innovation process.

\subsection{Conceptual foundations}

Nonaka puts forward two dimensions as conceptual basis for his model. The first dimension, called epistemological dimension, concerns the classic distinction between tacit and codified knowledge ${ }^{4}$. The second dimension, called ontological, underlines the importance of interaction between individuals as a vector of the creation of organizational knowledge. Basically, it is the individuals who create knowledge. The organization is only a trigger (more precisely an amplifier) for individual creation of knowledge:

\footnotetext{
${ }^{4}$ Nonaka suggests four modes for knowledge conversion to elaborate a sort of spiral of organizational knowledge creation. He suggests that the conversion between tacit and codified knowledge is a source for the creation of new knowledge. There are four types of conversion from tacit to tacit (socialisation), from codified to codified (combination), from tacit to codified (externalisation) and from codified to tacit (internalisation) (see Nonaka, 1994, for more details).
} 
"Organizational knowledge creation (...) should be understood in terms of process that "organizationally" amplifies the knowledge created by individuals, and crystallizes it as a part of the knowledge network of organization" (Nonaka, 1994, p. 17). Thus, individuals' knowledge is transformed and legitimized by a more or less formal organization.

The notion of commitment explains precisely the primacy of individuals in the process of knowledge creation. The staff of a firm is more or less committed in the process that, according to Nonaka, is similar to a redefinition of the world consistent with their perspectives. Three factors have an influence on individuals' commitment: intention, autonomy and fluctuation.

Intention is more than a mere state of mind, it is mainly the vector of an oriented or determined action. The purpose of this action, in evolutionary terms, is to ensure the "perenniality" of individuals and of the firm. Individuals' intention materializes in the elaboration of cognitive models in order to better apprehend the environment around them. This process implies value criteria, ways of judging information: "Without intention, it would be impossible to judge the value of the information or knowledge perceived or created" (Nonaka, 1994, p. 20).

The types of intention are obviously different according to the individual. An organization expresses a certain type of variety that it is useful to maintain in order to preserve flexibility in the acquisition and interpretation of information. In ensuring individuals' autonomy in their beliefs and judgment, the firm increases the probability of being able to acquire future opportunities, even those which it did not expect. This type of selforganization generates knowledge creation and favors synergy between individual varieties (Cohendet et al., 1998).

The ontological dimension of knowledge creation is also applied through the interaction of individuals with their environment. Environmental fluctuations, which are more or less chaotic and intermittent, infer a change in behavior, a review of representations of the outside world by the individuals. In this way, fluctuations constitute an incentive for knowledge creation according to the capacity of the individuals to understand and to adopt external changes, and also according to their intentions and their autonomy. In this context, the notion of rupture appears to be a determinant factor. Only through a certain type of rupture in relation to its habits, routines and beliefs can a firm evolve.

The ontological dimension assumes that there is communication between individuals who have often divergent and opportunistic interests. In an organization, the complementary and conflicting relationship between individuals is both productive and destructive (Cohen, 1984). In this respect, the hierarchical structure constitutes the order which ensures the maintenance and the absorption of the rewards of interaction.

\subsection{Innovation, interaction and information redundancy}

Nonaka $(1990,1995)$ defines the process of innovation as the product of the interaction between the need and the chance, the order and the disorder, the continuity and the discontinuity. In this context, the innovation is not only the result of a planned research program but also of uncertain events.

While building on case studies of Japanese firms, the author puts ahead the concept of information redundancy as a central element of the process of innovation in the firm. 
This concept is defined as an excess of information held by the set of the individuals. The interaction between the individuals ensures the mediatization of this necessary minimal information to understand the organization and to support the creation of organizational knowledge to innovate.

This form of learning by intrusion, to quote the wording of the author, makes it possible to reduce the specialties within the firm and to confer a transverse dimension to the innovation. The division of the labor takes here a new orientation in this direction, where at the same time the hierarchy and the bulk-heading of the specific activities of the individuals are reduced in order to allow the emergence of new organizational knowledge: "Rather, in what is also termed "a shared division of labor", every phase of innovation generation is loosely connected and overlaps, expanding and contracting with the unrestricted elasticity of diversity" (Nonaka, 1990, p. 29).

\section{The model}

In our model, which primarily consists in interpreting and adapting according to our problems the mathematical developments of Aubin (1997), we consider that a firm is described as a system with individuals. The state variable is defined by the individual contribution to the innovative performances of the firm. The innovative performances define the regulees of the system. The viability constraints are defined by the individual competencies to contribute to the innovative process of the firm.

In the first part, we describe different organizational structures according to viability behavior. This will be useful to compare, in a second part, different forms of dynamics and to demonstrate the hypothesis according to which interactions favor organizational knowledge creation and also improve innovative performances of the firm.

\subsection{Organization structures and behavioral viability of the firm}

To begin, we describe the firm's organizational structure in which individuals contribute to its innovative performances independently of individual competencies. This constraint will after be introduced to explain the viability problem which the firm faces. Then, we will introduce the interactions between individuals with the help of a connection matrix. Finally, we will describe an organizational structure which integrates the firm's innovative performances into individual behavior.

\subsubsection{Individuals' contributions to the firm's innovation}

Suppose a firm composed by $n$ individuals: $i=1, \ldots, n$.

In a situation where no constraint of competencies is imposed on the dynamics of individual contributions to the firm's innovative performances, the individual's dynamic behavior denoted by $x_{i}(t) \in Y$ at time $t \geq 0$ (with $Y$ a finite dimensional vector space such as $Y=R^{l}$ ) would be governed by a differential equation of the form, when $x_{i}$ represents the individual contribution $i$ :

$$
\text { Let } g_{i}: Y \rightarrow Y \text { then } i=1, \ldots, n \quad x_{i}^{\prime}(t)=g_{i}\left(\left(x_{i} t\right)\right)
$$


The preceding equation describes a sort of "isolated" learning (or self-taught) in the sense that an individual learns without interacting with others. Moreover, his contribution to the innovative performances of the firm is in this case independent of the competencies he may have.

In order to integrate the constraint of competencies into the individual contribution, we set $M \subset Y$ describe individuals' competencies such as:

$$
K \text { is the set of } x=\left(x_{1}, x_{2}, \ldots, x_{n}\right) \text { satisfying } h(x(t)) \in M
$$

For example, we consider that in our framework,

and

$$
\begin{gathered}
\forall t \geq 0, \sum_{i=1}^{n} x_{i}(t) \in M \\
y(t)=\sum_{i=1}^{n} x_{i}(t)=h(x(t))
\end{gathered}
$$

Each individual contributes to the firm's innovation in a way that the overall contribution satisfies all available competencies. This equation thus describes the individuals' behavior within the constraints of their competencies by supposing that the constraint, at collective level, is satisfied by independent behavior. We can also notice that this assumption is linked to the notion of redundancy of potential command puts forward by Nonaka which means that "the value of each part is potentially equal and arises from the nature of information in which the importance of each component contributes in its relation to the whole" (Nonaka, 1990, pp. 33-34).

\subsubsection{Interaction between individuals}

We will consider now an organizational structure in which individuals interact according to a coordination mode described with the help of a connection matrix.

If $X=Y^{n}$, we propose to connect the associated dynamics to the individual contributions through a connection matrix $W=\left(w_{i}^{j}\right) \in L(X, X)$ (where $L(X, X)$ is the vector space of matrices). We will consider that the dynamical evolution of the individual's contribution $i$ is governed by an evolution law such that:

$$
i=1, \ldots, n ; x_{i}^{\prime}(t)=\sum_{j=1}^{n} w_{i}^{j}(t) \cdot g_{j}\left(x_{j} t\right) \text { with } w_{i}^{j} \in L(Y, Y)
$$

The connection matrix determines the interaction between individuals who belong to the firm. This one is thus completely decentralized when the connection matrix is the identity matrix noted $\mathbf{1}$. Such an organizational structure, which could be defined as a hollow organization in which individuals have no relation, is a structure in which there is no learning from others.

In this organizational configuration, the viability problem consists in determining a regulation, which describes the consistency between individual behaviors according to their individual competencies.

The rule is that the total of individual contributions $y(t)=h(x(t))$ remains in the set of competencies, i.e. it remains inside $M$. 
When the total of contributions is almost to leave $M$, problems arise within the firm. This situation prevails if the velocity $y^{\prime}(t)$ "drives" individual contributions to leave $M$. In this case, the dynamic behavior of the firm is no longer viable. One solution consists in supposing $y^{\prime}(t)$ is tangent at $M$ and remains in the set of competencies. Mathematically, to clarify the viability theorem, the concept of contingent cone will be introduced (Aubin and Frankowska, 1990).

Definition 1. When $K$ is a subset of $X$ and $x$ belongs to $K$, the contingent cone $T_{K}(x)$ to $K$ at $x$ is the closed cone of elements $v$ such as:

$$
v \in T_{K}(x) \text { if and only if } \exists h_{n} \rightarrow 0+\text { and } \exists v_{n} \rightarrow v \text { such as } \forall n, x+h_{n} v_{n} \in K
$$

Thus, starting from $x$ in the direction of $v$, the individual's behavior approaches the boundary of $K$, but not too much, insofar, for a countable number of "steps" $h_{n}>0$ and approximate directions $v_{n}$, the point $x+h_{n} v_{n}$ lies in $K$. The contingent cone could be characterized in terms of distances: the contingent cone $T_{K}(x)$ to $K$ at $x$ is the closed cone of elements $v$ such that:

$$
\lim _{h \rightarrow 0+} \inf \frac{d(x+h v, K)}{h}=0
$$

We observe that

$$
\text { If } x \in \operatorname{Int}(K) \text {, then } T_{K}(x)=X
$$

and that if $K=\{\bar{x}\}, T_{\{\bar{x}\}}(\bar{x})=\{0\}$, we recall that a function $x():. I \rightarrow K$ is said to be viable if and only if

$$
\forall t \geq 0, x(t) \in K
$$

Then a differential viable function in $K$ satisfies

$$
\forall t \geq 0, x^{\prime}(t) \in T_{K}(x(t))
$$

We can also mention that the contingent cone coincides with the tangent space $T_{K}(x)$ of differential geometry when $K$ is a "smooth manifold". When $K$ is convex, we prove that the contingent cone coincides with the tangent cone $T_{K}(x)$ to $K$ at $x \in K$ of convex analysis, which is the closed spanned by $K-x$ :

$$
T_{K}(x)=\overline{U_{h>0} \frac{K-x}{h}}
$$

The tangent cone is convex. We prove that the contingent cone $T_{K}(x)$ is convex whenever $K$ is sleek at $x$ that the set-valued map $T_{K}($.$) is lower semicontinuous { }^{5}$ at $x$.

We can also apply the viability theorem. If $K=h^{-1}(M)$ where $h: X \rightarrow Y$ is a continuously differentiable map such that $h^{\prime}(x)$ is surjective and $M$ is closed and convex and then:

$$
T_{K}(x)=h^{\prime}(x)^{-1} T_{M}(h(x))
$$

\footnotetext{
${ }^{5}$ Cf. 2.1. Semicontinuous Set-Valued Maps and 5.1.2 Sleek Subsets in Aubin (1991, pp. 54 and 160).
} 
The contingent cone has been introduced to associate the individuals' behavior and the set of competencies with the regulation map.

Definition 2. The regulation map $R_{M}$ is the set-valued map:

$$
R_{M}(x)=\left\{W \in L(X, X) / h^{\prime}(x) W g(x) \in T_{M}(h(x))\right\}
$$

where $T_{M}(y)$ denotes the contingent cone to $M$ at $\mathrm{y}$.

The regulation map is elaborated by taking into consideration the consistency between individual contributions and their competencies. In our framework, the regulation map is seen as a sort of innovative behavioral programme of the firm. It is the formalization of behavioral rules of the viable firm.

\subsubsection{Innovative performances of the firm}

We couple now together the dynamics of individual contributions and innovative performances of the firm defined here as an effort (we subtract this effort from individual contributions), perceived as a message by individuals (regulee).

$$
i=1, \ldots, n \quad x_{i}^{\prime}(t)=g_{i}\left(x_{i}(t)\right)-p(t)
$$

with $p(t)$ defined as an effort by individuals and representing the innovative performances of the firm in order that viability constraints of the form

$$
\forall t \geq 0, h(x(t)) \in M \text { are satisfied. }
$$

We consider that individuals perceive innovative performances in a same way. The regulee represents here the notion of information redundancy, which is equal for all individuals.

The problem of viability consists in introducing a new regulation map taking into consideration the innovative performances defined here as a regulee.

The regulation map $\prod_{M}$ is the set-valued map such that:

$$
\forall x \in K, \prod_{M}(x)=\left\{p / h^{\prime}(x)(g(x)-p) \in T_{M}(h(x))\right\}
$$

The dynamics of the firm is viable if and only if individual contributions, according the to scheme of innovative performances, respect this regulation map.

These last two forms of organizational structure and their viability problem present different forms of process of dynamical contributions within the firm. We will now demonstrate that the dynamics according to the structure with interactions between individuals is the same as the dynamic according to the structure where individuals do not interact, but include innovative performances in behavior. More precisely, we demonstrate in the context of viability, that the evolution of innovative performances is minimal and identical as the evolution of contributions where individuals do not interact.

\subsection{Comparison of dynamical contributions}

Insofar as the simplest organization is described by the matrix identity, one can pose in a first stage the problem of the search for an organization which minimizes the distance $\|W-\mathbf{1}\|$. This distance indicates an indicator of connectionist complexity of the organization. 
In a more traditional way, we observe then the modes of organizations decentralized by the mechanisms of innovative performances. We also pose the problem to make evolve these innovative performances in order to guarantee viability and among these innovative performances, we choose the minimal norm $\|p\|$. We observe that the regulation by the innovative performances is a particular case of the regulation by the matrix of connections (to some extent summarized in the innovative performances).

One shows thus that the regulation by the simplest organization in the sense that the index of connectionist complexity is minimum, among all matrices regulating viable evolution, provides an evolution, which could be controlled by innovative performances. Moreover, the innovative performances have minimal norms.

Recall that in the case of regulation by innovative performances mechanisms, the regulation map is defined by:

$$
\forall x \in K, \prod_{M}(x)=\left\{p / h^{\prime}(x)(g(x)-p) \in T_{M}(h(x))\right\}
$$

The viability theorem involves that the innovative performances $p(t)$ regulating viable solutions are given by the regulation law:

$$
p(t) \in \prod_{M}(x(t))
$$

For determining feedback innovative performances, we can select some innovative performances of the regulation map like $\bar{\sigma}^{0}(x) \in \prod_{M}(x)$ with minimal norm. Viable solutions obtained with feedback innovative performances are the slow viable solutions.

When $B \in L(X, Y)$ is surjective, its orthogonal right inverse ${ }^{6}$ is equal to

$$
B^{+}=B^{*}\left(B B^{*}\right)^{-1}
$$

that we can supply $Y$ with the final norm $\mu^{B}$ defined by $\mu^{B}(z)=\left\|B^{+} z\right\|$ and that we denote by $\pi_{K}^{B}$ the projector of best approximation onto the closed subset $K$ for this final norm.

The unique solution $\bar{x}$ to the minimization problem ${ }^{7}$

$$
\inf _{B x \in K+v}\|x-u\|
$$

is equal to

$$
\bar{x}=u-B^{+}\left(\mathbf{1}-\pi_{M}^{B}\right)(B u-v) .
$$

Moreover, when $M$ is sleek (and in particular convex), then its tangent cones $T_{M}(y)$ are convex (Aubin and Frankowska, 1990). Since the polar cone to the contingent cone $T_{M}(y)$ is the normal cone $N_{M}(y)$, we can determine the solution to the minimization problem

$$
\inf _{B x \in T_{M}(y)+v}\|x-u\|
$$

is equal to

$$
\bar{x}=u-B^{+}\left(\mathbf{1}-\pi_{T_{M}(y)}^{B}\right)(B u-v)=u-B^{*} \pi_{N_{M}(y)}^{B^{*}}\left(B B^{*}\right)^{-1}(B u-v)
$$

${ }^{6}$ Cf. Definition 10.1.2 in Aubin (1997, p. 348).

${ }^{7}$ Cf. 10.1.1. Projections onto Inverse Images of Convex Sets in Aubin (1997, p. 348). 
where $\pi_{N_{M}(y)}^{B^{*}}$ denotes the projector onto the normal cone $N_{M}(y)$ when the dual $Y^{*}$ is supplied with the dual final norm $\left\|B^{*} q\right\|$.

Slow solutions to this dynamical system to our problem are given by

Proposition 1. Let assume that the map $g$ is continuous and bounded and that the competencies map $h$ is continuously differentiable and satisfies the uniform surjectivity condition:

$$
\forall x \in K, h^{\prime}(x) \text { is surjective } \& \sup _{x \in K}\left\|h^{\prime}(x)^{+}\right\|<+\infty
$$

and that $M$ is closed convex. Then the slow viable solution of the dynamic

$$
x^{\prime}(t)=g(x(t))-p(t)
$$

subjected to the viability constraints

$$
\forall t \geq 0, h(x(t)) \in M
$$

is the solution to the differential equation

$$
x^{\prime}(t)=g(x(t))-\varpi^{0}(x(t))
$$

where

$\bar{\varpi}^{0}(x)=h^{\prime}(x)^{+}\left(\mathbf{1}-\pi_{T_{M}(h(x))}^{h^{\prime}(x)}\right) h^{\prime}(x) g(x)=h^{\prime}(x)^{*} \pi_{N_{M}(h(x))}^{h^{\prime}(x)}\left(h^{\prime}(x) h^{\prime}(x)^{*}\right)^{-1} h^{\prime}(x) g(x)$

Proof. Under the criterion of lower semicontinuous condition, the regulation map $\prod_{M}$ is lower semicontinuous because the set-valued map $T_{M}($.$) is semicontinuous and the$ surjectivity assumption (6) implies assumption (11.2.1) of proposition (11.2.10) in Aubin (1997, pp. 363 and 368). Slow viable solutions exist and are obtained by the innovative performances $\Phi^{0}(x)$ of minimal norm among the innovative performances satisfying $h^{\prime}(x) p \in T_{M}(h(x))-h^{\prime}(x) g(x)$. Proposition (10.1.4.) in Aubin (1997, p. 349) implies formula (7).

We will now compare the dynamics of contributions dictated by the connection matrices which describe coordination between individuals in a firm with those obtained by independent contributions to innovative performances.

We compare the dynamics $x^{\prime}(t)=g(x(t))-p(t)$ which respects minimal cognitive constraints $\forall t \geq 0, \sum_{i=1}^{n} x_{i}(t) \in M$, to that whereby individuals are connected by a connection matrix $W \stackrel{i=}{=}\left(w_{i}^{j}\right) \in L(X, X)$ which can be written as follows:

$$
x^{\prime}(t)=W(t) g(x(t))
$$

We propose to demonstrate that the dynamics linked to $x^{\prime}(t)=g(x(t))-p(t)$ and that linked to $x^{\prime}(t)=W(t) g(x(t))$ are identical.

Whenever $p \in X^{*}$ (dual of the matrix $X$ ) and if $y \in Y$, we denote by $p \otimes y \in L(X, Y)$ (tensor product ${ }^{8}$ ) the rank one linear operator defined by $x \rightarrow(p \otimes y)(x)=\langle p, x\rangle y$, the matrix of which is

$$
\left(p^{i} y_{j}\right)_{i}=1, \ldots, m, m
$$

${ }^{8}$ Cf. Tensor Products of Linear Operators in Aubin (1997, p. 353). 
Both finite dimensional vector spaces $X$ and $Y$ are identified with their duals. We observe then:

Proposition 2. Let $\Gamma: X \rightarrow X$ be any continuous map such that

$$
\inf _{x \in K}\langle\Gamma(x), g(x)\rangle>0
$$

Then a connection matrix of the form

$$
W(x)=\mathbf{1}-\frac{\Gamma(x)}{\langle\Gamma(x), g(x)\rangle} \otimes \varpi(x)
$$

where the values $\varpi_{i j}(x)$ equal to

$$
\varpi_{i j}(x)=\delta_{i, j}-\frac{\Gamma(x)_{i} \varpi(x)_{j}}{\langle\Gamma(x), g(x)\rangle}
$$

belongs to $R_{M}(x)$ if and only if $\varpi(x)$ belongs to $\prod_{M}(x)$ and then the viable solutions to $x^{\prime}=W(x) g(x)$ and $x^{\prime}=g(x)-\bar{\omega}(x)$ are identical.

Proof. We observe that

$$
W(x) g(x)=g(x)-\frac{\langle\Gamma(x), g(x)\rangle}{\langle\Gamma(x), g(x)\rangle} \varpi(x)=g(x)-\varpi(x)
$$

Then the differential equation $x^{\prime}=W(x) g(x)$, and $x^{\prime}=g(x)-\bar{\omega}(x)$ are the same.

If $W(x) \in R_{M}(x)$ then $h^{\prime}(x) W(x) g(x)=h^{\prime}(x) g(x)-h^{\prime}(x) \varpi(x) \in T_{M}(h(x))$ and the viability conditions are also the same.

The regulation by connection matrices of the form

$$
W(x)=\mathbf{1}-\frac{g(x)}{\|g(x)\|^{2}} \otimes \varpi(x)
$$

is the same as an innovative performances mechanism whenever $g(x) \neq 0$ for each $x \in K$.

In this way, the problem of maximal decentralization is then to find connection matrices as close as possible to the identity matrix.

Proposition 3. Suppose $g$ is a continuous and bounded map and the map of individual competentices $h$ is continuously differentiable and satisfies the uniform surjectivity condition:

$$
\forall x \in K, h^{\prime}(x) \text { is surjective and } \sup _{x \in K} \frac{\left\|h^{\prime}(x)^{+}\right\|}{\|g(x)\|}<+\infty
$$

Suppose that $M \subset Y$ is a closed convex subset. Then the set of competencies $K=h^{-1}(M)$ is viable for equation (8), denoting the organizational structure when individuals are interconnected if and only if for each $x \in K$, the image $R_{M}(x)$ of the regulation map is not empty.

The evolution resulting from the maximal decentralization subject to the viability constraint:

$$
\forall t \geq 0, h(x(t)) \in M
$$


is governed by the differential equation:

$$
x^{\prime}(t)=W^{0}(x(t)) g(x(t))
$$

where

$$
\begin{aligned}
& W^{0}(x)=\mathbf{1}-\frac{g(x)}{\|g(x)\|^{2}} \otimes h^{\prime}(x)^{+}\left(\mathbf{1}-\prod_{T_{M}(h(x))}^{h^{\prime}(x)}\right) h^{\prime}(x) g(x) \\
& =\mathbf{1}-\frac{g(x)}{\|g(x)\|^{2}} \otimes h^{\prime}(x)^{*} \pi_{N_{M}(h(x))}^{h^{\prime}(x)^{*}}\left(h^{\prime}(x) h^{\prime}(x)^{*}\right)^{-1} h^{\prime}(x) g(x)
\end{aligned}
$$

The slow viable solutions to the dynamics $x^{\prime}(t)=g(x(t))-p(t)$ and $x^{\prime}(t)=$ $W(t) g(x(t))$ are the same. In others words, the dynamics of a decentralized organizational structure (individuals do not interact) is equivalent to the dynamics of an organizational structure where the innovative performances of the firm are included into the individual contributions and are minimal.

Proof. The regulation map could be denoted as following:

$$
R_{M}(x)=\left\{W \in L(X, X) /\left(g(x) \otimes h^{\prime}(x)\right) W \in T_{M}(h(x))\right\}
$$

Then the viable solutions are regulated by the regulation law:

$$
(g(x(t))) \otimes\left(h^{\prime}(x)(t)\right) W(t) \in T_{M}(h(x))
$$

Insofar as the map $g(x) \otimes h^{\prime}(W x)$ is surjective from the space $L(X, X)$ of connection matrices to the individual competencies space $Y$, then

$$
\left\|\left(g(x) \otimes h^{\prime}(x)\right)^{+}\right\|=\frac{\left\|h^{\prime}(x)^{+}\right\|}{\|g(x)\|}
$$

Then, assuming that $R_{M}$ is lower semicontinuous, the viable connection matrices near the identity matrix are the solution of $W^{0}$ minimizing the distance $\|W-\mathbf{1}\|$ among the connection matrices satisfying the viability constraint.

According to the theorem of orthogonal projections on subset of matrices ( $c f$. theorem 10.3.6. in Aubin (1997) p. 358), the map $g(x) \otimes h^{\prime}(W x)$ is surjective from the space $L(X, X)$ of connection matrices to the individual competencies space. The solution is given by (10).

\section{Conclusion}

We showed that the dynamics of innovative behavior where agents interact is the same as the dynamic where the innovative performances of the firm are included in individual's contribution. This analysis provides a strong analytical framework to the conceptual work proposed by Nonaka.

Certain points could be developed, in particular the analysis of viability within the framework of two qualification levels (individual and organizational) and the questions 
related to the interpretation of core competencies of the firm according to the viability kernel ( $c f$. Munier 1999). Other points could also be investigated. The determination of selection mechanisms for the behavior of the firm constitutes an important question. It would be also interesting to treat the interactions between the individuals by introducing a matrix of connection at the level of competencies. All these questions could be analyzed with viability theory and allow to further consolidate the relevance of this mathematical theory to analyze economic issues.

\section{References}

Aubin J.-P. (1991) Viability theory. Birkhäuser, Boston, Basel.

Aubin J.-P. (1997) Dynamic economic theory, a viability approach, Studies in Economic Theory 5, Springer, Berlin.

Aubin J.-P., Foray D. (1998) The emergence of network organizations in processes of technological choice: A viability approach. In: Cohendet P., Llerena P., Stahn H., Umbhauer G. (Eds.), The economics of networks, interaction and behavior. Springer, Berlin.

Aubin J.-P., Frankowska H. (1990) Set-valued analysis. Birkhaüser, Boston, Basel, Berlin.

Bebernes J.W., Schuur J.D. (1970) The Wazewski topological method for contingent equations, Annali di Mat. Pura ed Appli 87, pp. 271-280.

Cohen M.D. (1984) Conflict and complexity: Goal diversity and organizational search effectiveness, American Political Science Review 78, pp. 435-454.

Cohendet P., Kern F., Mehmanpazir B., Munier F. (1998) Routines, structure of governance and knowledge-creating processes. In: Lesourne J., Orléan A. (Eds), Advances on self-organization and evolutionary theory. Economica, Paris .

Haddad G. (1981), Monotone trajectories of differential inclusions with memory, Israel Journal of Mathematics 39, pp. 83-100

Munier F. (1999) Taille de la firme et innovation: approches théoriques et empiriques fondées sur le concept de compétences. Thèse de doctorat, Université Louis Pasteur, Strasbourg 1.

Nonaka I. (1990) Redundant, overlapping organization: A Japanese approach to managing the innovation process, California Management Review, Spring, pp. 27-38.

Nonaka I. (1994) A dynamic theory of organizational knowledge creation, Organisation Science 5, No. 1, pp. 4-37.

Nonaka I. (1995) Managing innovation as an organizational knowledge creation process. In: Allouch J., Pogorel G. (Eds.), Technology management and corporate strategies: A tricontinental perspective. Elsevier Science B.V., pp. 73-109.

To access this journal online: www.edpsciences.org 